\title{
ORCHIDS SMARTER THAN SCIENTISTS - AN APPROACH TO ONCIDIINAE (ORCHIDACEAE) TAXONOMY
}

\author{
STIG DALSTRÖM \\ Research Associate, Marie Selby Botanical Gardens, 811 South Palm Avenue, Sarasota, FL 34236, U.S.A. \\ sdalstrom@selby.org
}

When initiating a project in plant taxonomy it is necessary to carefully examine the type specimen for each taxon that has been described. Just as important, however, is to explore the natural habitats of the plants because that is where the dynamics occur, which is the logical source for the necessary data. It is in nature where we best can develop an understanding of what a "species" really is and its role in the environment. Another fact to remember is that a previously described "species" does not necessarily constitute a good natural species. Specific and generic concepts can differ drastically between scientists even regarding the same group of plants (e.g., Dalström 2001b versus Christenson 2002).

It appears generally accepted that the primary basis of evolution in all organisms is mutation. Through minor and frequent mutations an isolated population that does not exchange genetic information with other populations can keep even initially insignificant genetic changes inside their own group. Over many new generations of plants they will eventually have time to amplify the changes and become distinct. Another possible source of new taxa is through natural hybridization where the offspring become so drastically different from the parents that they will not cross-pollinate with any of them. The hybrids therefore "need" to find a new pollinator or develop some other isolating barrier to ensure a separate evolutionary path. It has been possible to identify many natural hybrids in Odontoglossum Kunth that were originally described as species by crossing the suspected parent species under artificial conditions (Rolfe 1893). Some species are more promiscuous than others and one of the worst is Odontoglossum crispum Lindl. Over one hundred "varieties" [color forms] have been named (Bockemühl 1989), which demonstrates the incredible variability and popularity of this species alone.
Many of the color forms are probably the results of cross-pollination with other species.

I define a species as a group of plants that look alike regarding taxonomically important features and which produce offspring with the same important features as the parents. I distinguish a species by one or more unique important features, or a unique combination of features, which constitutes the species profile. A species profile generally, but not exclusively, consists of features that are associated with the pollination apparatus (column and lip relation in Oncidiinae) while a genus profile can consist of many other features as well, such as vegetative or anatomical differences. A genus is a selected group of species, which share a unique combination of features that distinguish them from plants in other genera. A subtribe is a group of selected genera, which share some unique features, or combination of features that make them different from other subtribes, etc.

I consider the pollination of the flower the most significant part of the speciation process. Therefore, we need to study the design of the pollination apparatus to distinguish the various species. Some visual and obvious characteristics of the flower morphology, such as a larger or smaller callus on the lip, variation in color, etc., are obvious features and may initially seem important. They can also be the result of changes in the environment, however, such as cooler temperatures, brighter light or dryer air, which affect the general health of the plant. Plants in the subtribe Oncidiinae demonstrate a notorious ability to change the flower appearance due to an environmental impact (stress). Naturally, the size and age of a plant also matters. I call this ability a plant's natural plasticity, which is different from a plant's natural variability. Compared with humans one could say that 
"plasticity" represents our habit of making different faces depending on our mood, as opposed to how different individuals appear in length, nose shape or hair color, regardless of the mood (= variability). In Odontoglossum many of the traditional distinguishing features between species are "cosmetic" due to variability and plasticity and do not really tell us much about the species profile. Some species descriptions are also based on cultivated and stressed plants, which can produce flowers with deviating shapes. Obviously, different species of the same genus may also have strikingly different plant habits and anatomy in addition to floral differences. In Odontoglossum, however, the species are so vegetatively similar that they look pretty much the same without flowers. They may differ in mature size and shape but a young plant of a large species looks very much like a large plant of a small species.

Most species of Odontoglossum were described during the nineteenth century by taxonomists with very little or no natural experience of the plants or their habitats, which led to descriptions based on very limited knowledge. As a metaphor we can use the Andes hidden in mist. As the mist evaporates the individual peaks begin to emerge. This is how orchid species often are perceived, as isolated and distinct entities separated by shape, size or color. As the mist continues to evaporate the peaks connect via intermediate ridges and eventually turn into one continuous mountain range. Orchid species that originally appear distinct often become connected when more plant material is examined from intermediate localities. Many Odontoglossum species are not distinct entities but rather belong to a complex of similar taxa. In some cases the members of a complex have satisfactory profiles to justify a specific status. In other cases, they connect with each other by intermediate forms. If the complex is too blurry I prefer to treat the entire complex as a superspecies, which refers to a level slightly "above" a more traditional species concept. A superspecies consists of subspecies, which refers to a level slightly "below" species, and are geographically restricted populations of plants with a shared sub-profile that differs from other subspecies. A subspecies is morphologically relatively consistent within the population and does not generally mix with other sub- species of the same superspecies. In my vocabulary, the term "variety" refers to individual plants that share some deviating features, such as a lack of pigment, with individual plants in other populations, which has little taxonomic significance.

Traditionally, the flower shape has dominated the basis for Oncidiinae taxonomy. The angle between the column and the lip has played a major role in how we classify plants on a generic level, while features such as plant shape and habit, micro-morphology of the pollinarium and plant anatomy have been largely ignored. Vegetatively different plants have ended up in the same genus because they have flowers with a similar angle between the lip and the column. One of the problems with this approach is that the angle is not a definite feature, but rather a gliding scale from the lip and column being parallel, to $180^{\circ}$ apart, or more. Both the lip and the column also show a great diversity and irregularity in shape and size, which makes it difficult to measure the angle in a consistent way. It is actually rather strange that so much attention has been paid to a few floral features when the vegetative parts of the plants can show a great differentiation in shape and habit. On a specific level, however, it seems logical to assume that the most important aspect of a plant's life is the reproduction phase. Therefore, the pollination strategy and dynamics must be intimately involved with a species evolution and identity. Consequently, the taxonomically important features are those that concern the pollination apparatus (column and lip relation in Odontoglossum), which need to be rather stable.

There seem to exist two antagonistic "forces" in nature, which concern the reproduction of flowering plants. One force encourages morphological stability to enable a species to develop some kind of consistent inter-action with a particular pollinator. The reason why cross-pollination (the exchange of pollen between two separate individual plants, or clones) is preferable to self-pollination is that the exchange of genetic information increases variability. This, in turn, allows a wider adaptation to possible changes in the environment. If the flowers kept changing continuously, however, the result would be a genetic and morphological chaos, and a probable extinction due to a lack of fitting pollinators. The extreme conse- 
quence of a too stable morphology, on the other hand, would result in an inability to adapt to changes in the environment and to new pollinators, which inevitably also would lead to extinction. A balance between genetic stability and variability through mutations, natural hybridization and plasticity, seems to be the successful choice. It is simple to discern a successful strategy versus an unsuccessful one in Odontoglossum because the plants either exist commonly or not, and the majority of Odontoglossum species display a great variability. It seems logical that plants with a variable gene-code have a higher survival rate in an unstable environment, than do plants with a more fixed genetic constitution. The Andean region of South America provides a constantly changing environment due to volcanic eruptions, earthquakes, landslides and floods, and more recently, man's occurrence on the stage. In a longer perspective, fluctuation in temperature has caused periods of glaciations as well. Consequently, a variable habitat would also favor a rapid and diverse speciation, which seems to be the case in the Andes.

The flowers of Odontoglossum are considered rewardless. They do not offer any kind of obvious reward, such as nectar or oil for the pollinator. Theoretically, a pollinator may visit a seemingly rewardless flower for some other rewarding reasons, such as absorbing a scent or possibly to deposit eggs on the flower, which in turn may feed the larvae. No such observations are documented, however. In order to encourage the pollinator to re-visit a rewardless flower the plant has to develop a deceit pollination strategy, and this seems to be done with the help of variable and plastic features, or cosmetics. Examples of cosmetics are coloration patterns, variation in the callus size and shape, width of sepals and petals, and possibly a variation in odors (e.g. the different parts of the flower of Odontoglossum hallii Lindl. have different smells). A certain degree of natural hybridization also seems favorable to an increased variability as long as the pollination strategy is not broken up and becomes chaotic. In conclusion, we have certain features, which vary not only between different individual plants but also between individual flowers on the same raceme and are assumed to be deceptive. They convince the pollinator to make yet another try in its quest for whatever reward it is searching for. Cosmetic features are here called taxonomically unimportant and are unreliable for distinguishing species. Features, such as the pollination apparatus, that "design" the flower to accommodate particular pollinators are taxonomically important features. These cannot change too much because it would lead to a morphological chaos with consequent pollination problems. Unfortunately, what works to fool pollinators also fools scientists. Only by visiting many populations and studying large quantities of plants can we see through this deception and discern natural species.

When we fully understand the evolutionary dynamics in a small group of plants, such as Odontoglossum, we discover a valuable key to the understanding of how other organisms evolve on Earth as well. Repetitive speciation patterns on many levels in plants have lead to the development of superficially similar flowers based on similar pollination strategies. Using the flower morphology exclusively for taxonomic treatments may seem convenient and user-friendly but can be misleading and confusing at the same time because it does not necessarily relate to the natural relationship between species and genera. In contrast, with the recent development of DNA sequence analysis we have found another key to the classification of troublesome plants. Although still at an experimental, initial stage of its development, it is now possible to learn about the genetic relationships of the plants. This can be very useful in some cases, such as the delineation and separation of Cyrtochilum from allied genera (Dalström 2001a). Unfortunately it also creates a new dimension of problems when we realize that morphologically different looking plants can be closely related, and viceversa, thus breaking up traditional classification and creating a very unpractical system. In other words, with a classification system based solely on DNA analysis, you may have to know the plant before you can identify it, because there are no reliable and visible indications where it belongs.

\section{Literature Cited}

Bockemühl, L. 1989. Odontoglossum, a Monograph and Iconograph. Brücke-Verlag Kurt Schmersow, Hildesheim. Germany. 
Christenson, E. A. 2002. Cochlioda - A taxonomic treatment of this New World genus. Orchids 2(110-121).

Dalström, S. 2001a. A synopsis of the genus Cyrtochilum (Orchidaceae; Oncidiinae): Taxonomic reevaluation and new combinations. Lindleyana 16(2): 56-80.
Dalström, S. 2001b. New species and combinations in the Oncidiinae (Orchidaceae) and a synopsis of the Cochlioda clade. Selbyana 22(2): 135-145.

Rolfe, R. A. 1893. Hybrid Odontoglossums. Orch. Rev. 1(5): 142 .

Stig Dalström is a free-lance botanical artist, and Research Associate of the Marie Selby Botanical Gardens, Sarasota, Florida, US. He specializes in Oncidiinae taxonomy, particularly the Andean species of Cochlioda, Cyrtochilum, Oncidium and Odontoglosum. 